\title{
Prevention of device-related tissue damage during percutaneous deployment of tissue-engineered heart valves
}

\author{
U.A. Stock, MD, ${ }^{a, b, c}$ I. Degenkolbe, ${ }^{a, b}$ T. Attmann, MD, ${ }^{d}$ K. Schenke-Layland, PhD, ${ }^{e}$ S. Freitag, PhD, ${ }^{f}$ and G. Lutter, MD ${ }^{d}$
}

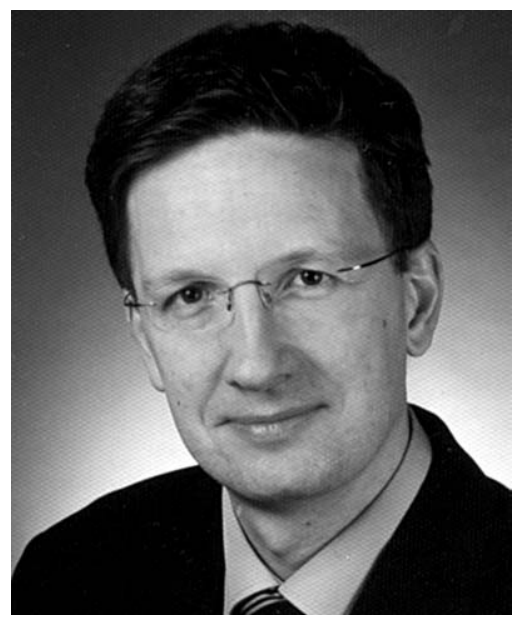

Dr Stock

Background: Endovascular application of pulmonary heart valves has been recently introduced clinically. A tissue-engineering approach was pursued to overcome the current limitations of bovine jugular vein valves (degeneration and limited longevity). However, deployment of the delicate tissue-engineered valves resulted in severe tissue damage. Therefore the objective of this study was to prevent tissue damage during the folding and deployment maneuver.

Material and Methods: Porcine pulmonary heart valves, small intestinal submucosa, and ovine carotid arteries were obtained from a slaughterhouse. After dissection and antimicrobial incubation, the valves were trimmed (removal of sinus and most of the muscular ring) to fit into the deployment catheter. The inside (in-stent group, $\mathrm{n}=$ 6) or outside (out-stent group, $n=6$ ) of a nitinol stent was covered by an acellular small intestinal submucosa, and the valves were sutured into the stent. The valves were folded, tested for placement in the deployment catheter, and decellularized enzymatically. Myofibroblasts were obtained from carotid artery segments and seeded onto the scaffolds. The seeded constructs were placed in a dynamic bioreactor system and cultured for 16 consecutive days. After endothelial cell seeding, the constructs were folded, deployed, and processed for histology and surface electron microscopy.

From the Department of Cardiothoracic and Vascular Surgery, ${ }^{\text {a }}$ Friedrich-SchillerUniversity, Jena, Germany; the Department of Medical Physics and Biophysics, ${ }^{\text {b }}$ Charite University, Berlin, Germany; the Department of Cardiovascular Surgery, Heart Center Brandenburg, Bernau/Berlin, Germany; the Department of Cardiovascular Surgery ${ }^{\mathrm{d}}$ and the Department of Medical Statistics, ${ }^{f}$ Christian-Albrechts-University, Kiel, Germany; and the Cardiovascular Research Laboratories, ${ }^{\mathrm{e}}$ David Geffen School of Medicine, UCLA, Los Angeles, Calif.

Supported by grants from the German Research Council Deutsche Forschungsgemeinschaft (STO359/2-3, STO359/4-1 S.U.A., LU 367/4-1 L.G. and SCHE 701/ 2-1 S-L. K.).

Received for publication Oct 31, 2005; revisions received Jan 15, 2006; accepted for publication Jan 24, 2006.

Address for reprints: Ulrich A. Stock, MD, Department of Medical Physics and Biophysics, University Hospital Charité, Invalidenstrasse 42, 10098 Berlin, Germany (E-mail: ulrich.stock@charite.de).

J Thorac Cardiovasc Surg 2006;131:1323-30 $0022-5223 / \$ 32.00$

Copyright (C) 2006 by The American Association for Thoracic Surgery

doi:10.1016/j.jtcvs.2006.01.053

Results: The valves opened and closed competently throughout the entire dynamic culture. Surface electron microscopy revealed an almost completely preserved tissue in the in-stent group. Stents covered with small intestinal submucosa on the outside, however, showed severe damage.

Conclusion: This study demonstrates that small intestinal submucosa covering of the inside of a pulmonary valved stent can prevent stent strut-related tissue damage.

$\mathrm{P}$ ulmonary valve regurgitation with late right ventricular dysfunction after repair of tetralogy of Fallot is a well-known entity. Current treatment options include right ventricular outflow tract (RVOT) reconstruction by means of either valved conduit surgery ${ }^{1}$ or percutaneous endovascular valve implantation. ${ }^{2}$ Even so, the latter concept represents a tremendous benefit for the patients because it avoids a redo operation; the currently used bovine jugular vein valves are glutaraldehyde-fixed xenogeneic heart valves likely to be prone to degeneration and will eventually require reinterventions. Tissue engineering might offer a potential pathway to overcome the present limitations of heart valve substitutes in general. ${ }^{3}$ Tissue-engineered valves will have unique characteristics of viability and ability to grow and remodel. Preliminary results, however, revealed that the folding and deployment maneuver of the extremely fragile and delicate tissue-engineered valves resulted in severe cell abrasion (Stock et al, unpublished data, 2005). Because this damage is caused by the stent struts itself, the objective of this study was to develop a strategy to prevent tissue damage during the folding and deployment maneuver by shielding the valve from the stent struts. 


\author{
Abbreviations and Acronyms \\ $\mathrm{EC}=$ endothelial cell \\ EDTA $=$ ethylenediamine tetraacetic acid \\ $\mathrm{MF}=$ myofibroblast \\ PBS = phosphate-buffered saline \\ PS = penicillin-streptomycin \\ RVOT $=$ right ventricular outflow tract \\ SIS $=$ small intestinal submucosa
}

\section{Materials and Methods}

The general approach to cell isolation, culture, and seeding, as well the basic decellularization procedure, was described in detail previously ${ }^{4}$; however, several modifications were required and applied. This study conformed to the "Guide for the care and use of laboratory animals" published by the US National Institutes of Health (publication no. 85-23, revised 1996).

\section{Cell and Tissue-culture Medium}

Standard cell and tissue-culture medium was Dulbecco's modified Eagle's medium (Invitrogen, Carlsbad, Calif) supplemented with $10 \%$ fetal calf serum (Biochrom AG, Berlin, Germany), $1 \%$ penicillinstreptomycin (PS; 10,000 U/10 mg/mL, Invitrogen), and $1.5 \mu \mathrm{g} /$ $500 \mathrm{~mL}$ recombinant human fibroblast growth factor, basic (Promega GmbH, Mannheim, Germany).

\section{Cell Isolation and Culture}

Six- to 9-cm-long segments of ovine carotid arteries were obtained from approximately 6-month-old slaughterhouse lambs by using a clean surgical technique. The arteries were stored in Dulbecco's modified Eagle's medium with fetal calf serum and PS at $4{ }^{\circ} \mathrm{C}$ and processed within 60 minutes. Endothelial cells (ECs) were obtained by means of a collagenase instillation technique for 20 minutes at $37^{\circ} \mathrm{C}$ and $5 \% \mathrm{CO}_{2}(0.2 \%$ collagenase type $\mathrm{A}$ [Roche Diagnostics GmbH, Mannheim, Germany] in phosphate-buffered saline [PBS; Invitrogen]) and cultured in $0.1 \%$ gelatin-precoated (gelatine type A; Sigma-Aldrich Chemie GmbH, Munich, Germany) tissue-culture flasks (BD Falcon; BD Biosciences, San Jose, Calif). The remaining vessel wall segments were minced into $1-\mathrm{mm}^{2}$ pieces, distributed on Petri dishes (Nunc GmbH, Wiesbaden, Germany) and cultured in cell-culture medium. After migration of the myofibroblasts (MFs) on the culture dishes (5-9 days after harvest), cells were cultured in gelatin-precoated flasks. Both cell populations were incubated at $37^{\circ} \mathrm{C}$ and $5 \% \mathrm{CO}^{2}$ and serially passaged with trypsin-ethylenediamine tetraacetic acid (EDTA) in Hanks' buffered saline solution (Invitrogen). Approximately $28 \pm$ 3 days were needed to obtain 15 confluent T75 tissue-culture flasks.

\section{Tissue Harvest}

Hearts and small intestines of pigs (90-120 kg weight) were obtained under clean conditions from a local slaughterhouse within 15 minutes of slaughter and immediately transferred in Hanks' buffered saline solution (Biochrom AG) to the laboratory. Pulmonary valves were excised and freed of adherent fat and most of the myocardium, leaving only a thin ridge of subvalvular muscle tissue

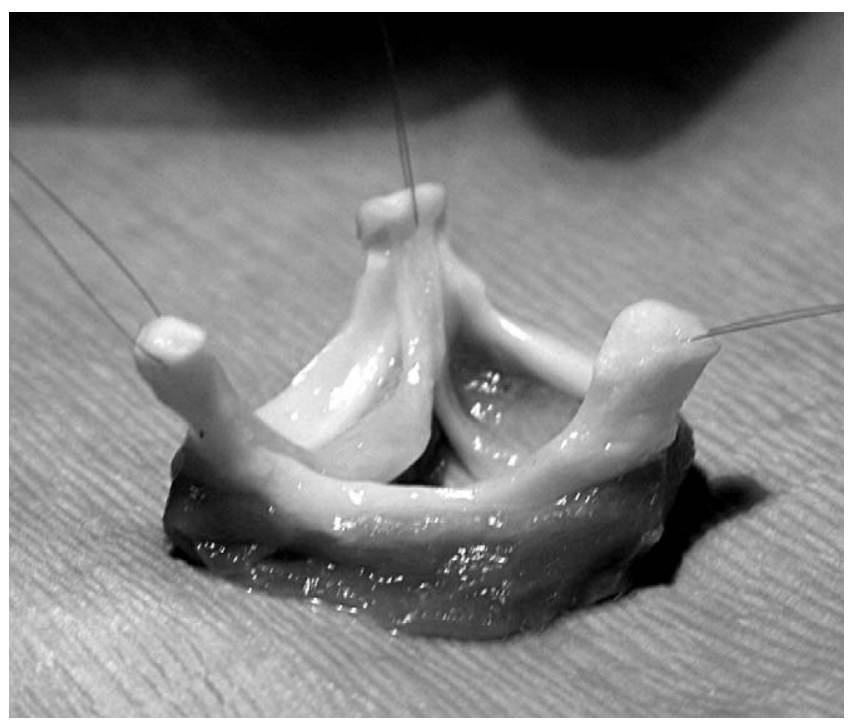

Figure 1. Scalloped pulmonary heart valve, leaving only the commissures and leaflets.

and the pulmonary artery (Figure 1). Six porcine pulmonary valves were dissected to match the size of the stent and the valve for each stented valve. The valve diameter was determined with a Hegar sizer before sinus removal and muscular trimming. Valves with a diameter of $24 \mathrm{~mm}$ were chosen for implantation into the $24-\mathrm{mm}$ stent. Segments of porcine small intestine (approximately $20 \mathrm{~cm}$ in length) were cleaned by thoroughly washing with PBS (Invitrogen), followed by incubation in povidone-iodine solution (Mundipharma, Limburg, Germany) solution for 15 minutes. Decontaminated segments were repeatedly washed with PBS, and the mucosa and any connective tissue were removed mechanically. ${ }^{5-7}$ The remaining small intestinal submucosa (SIS) was transferred into PBS containing an antibiotic solution (1.2 mg of amikacin, 3 $\mathrm{mg}$ of flucytosin, $1.2 \mathrm{mg}$ of vancomycin, $0.3 \mathrm{mg}$ of ciprofloxacin, and $1.2 \mathrm{mg}$ of metronidazol in $1 \mathrm{~mL}$ of aqua ad inject) and stored at $4^{\circ} \mathrm{C}$ for 48 hours.

\section{Stent Characteristics and Design}

Radially self-expanding nitinol stents (Nitinol Devices \& Components Inc, Fremont, Calif) were used in the present study. The stent wires are soft and highly malleable, molding 2 ranks of 14 rhombs each. Bare stents were $28 \mathrm{~mm}$ in length and $24 \mathrm{~mm}$ in diameter when fully expanded at $37^{\circ} \mathrm{C}$.

The stent is sterilized in $98 \%$ alcohol for 60 minutes with subsequent PBS washing and dried overnight with UV light in a laminar flow box.

\section{Valved Stent Construction}

The inside (in-stent group, $\mathrm{n}=6$ ) or outside (out-stent group, $\mathrm{n}=$ 6) of a nitinol stent was covered with an acellular porcine SIS, and the valves were sutured into the covered stent with interrupted 7-0 monofilament sutures (Prolene; Ethicon, Norderstedt, Germany; Figure 2). 


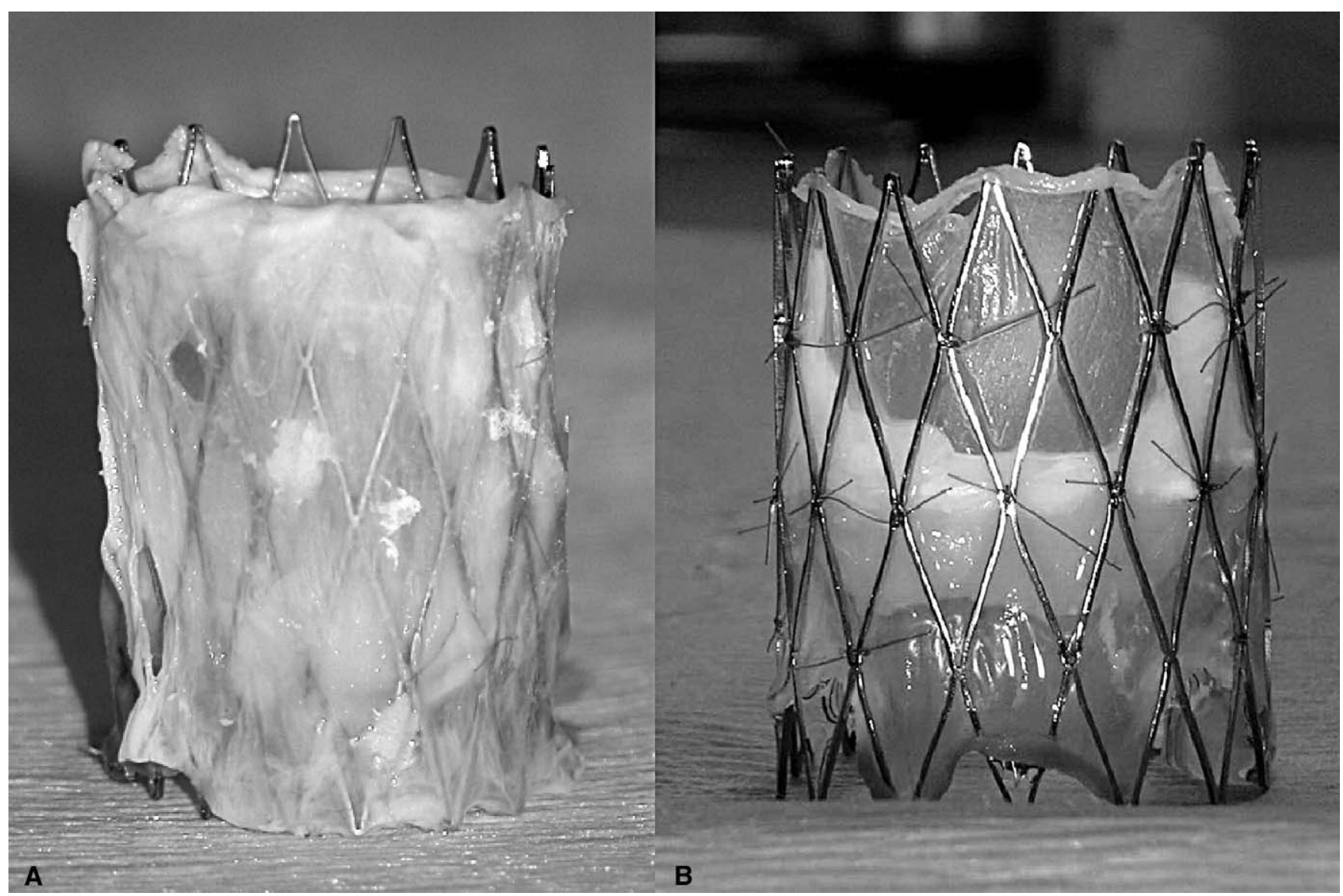

Figure 2. A, Out-stent construct with SIS surrounding the stent, resulting in a direct contact of the valve with the stent struts. B, In-stent construct with SIS located in between the nitinol stent and the scalloped valve.

\section{Decellularization Procedure}

The entire construct (stent with SIS and implanted valve) was washed for 30 minutes at room temperature in povidone-iodine solution and sterile PBS, followed by another overnight incubation at $4{ }^{\circ} \mathrm{C}$ in an antibiotic solution $(1.2 \mathrm{mg}$ of amikacin, 3 $\mathrm{mg}$ of flucytosin, $1.2 \mathrm{mg}$ of vancomycin, $0.3 \mathrm{mg}$ of ciprofloxacin, and $1.2 \mathrm{mg}$ of metronidazol in $1 \mathrm{~mL}$ aqua ad inject). After this decontamination procedure, the valves were placed in a solution of $0.05 \%$ trypsin and $0.02 \%$ EDTA (Biochrom AG) at $37^{\circ} \mathrm{C}$ and $5 \% \mathrm{CO}_{2}$ for 12 hours during continuous 3-dimensional shaking. After removal of the trypsin-EDTA, the constructs were washed with PBS for another 24 hours to remove residual cell detritus. The decellularized matrices were immediately processed for subsequent cell seeding.

\section{Reseeding and Dynamic Culture Procedure}

After cell expansion to a total quantity of approximately $3 \times$ $10^{7}$ cells, MFs were trypsinized, resuspended in culture medium, and seeded onto the decellularized pulmonary valves in a sequential seeding process that involved 2 days of static seeding on the inner and outer surface. Subsequently, the valves were placed in a pulsatile flow system (bioreactor, Figure 3) and exposed to defined physical signals with a fixed frequency (1 $\mathrm{Hz}$ ) and pressure conditions ( $3 \mathrm{~L} / \mathrm{min}$ and $60 / 40 \mathrm{~mm} \mathrm{Hg}$ ) over a time period of 16 days. A final coating with ECs $\left(9 \times 10^{6}\right.$ cells) for 2 days under static conditions, which allows the cells to form sediment on the valve wall and the leaflets, completed the reseeding. The entire culture period was conducted in a standard incubator at $37^{\circ} \mathrm{C}$ and $5 \% \mathrm{CO}_{2}$. PS supplement $(5 \mathrm{~mL})$ was added every 4 days.

\section{Folding and Deployment Maneuver}

Seeded and cultured valved stents were manually folded and inserted into a 22F modified commercially available catheter. Folding was achieved by means of uniform pressure application of the thumb and index, middle, and ring fingers of both hands. After a learning curve, all valves in this study were folded and pushed into the deployment catheter in one step by one investigator (D.I.). After 5 minutes of folding, the valved stent was deployed in vitro. They were placed back in the bioreactor and tested for competent opening and closing by using PBS. Immediately afterward, they were analyzed macroscopically and histologically. 


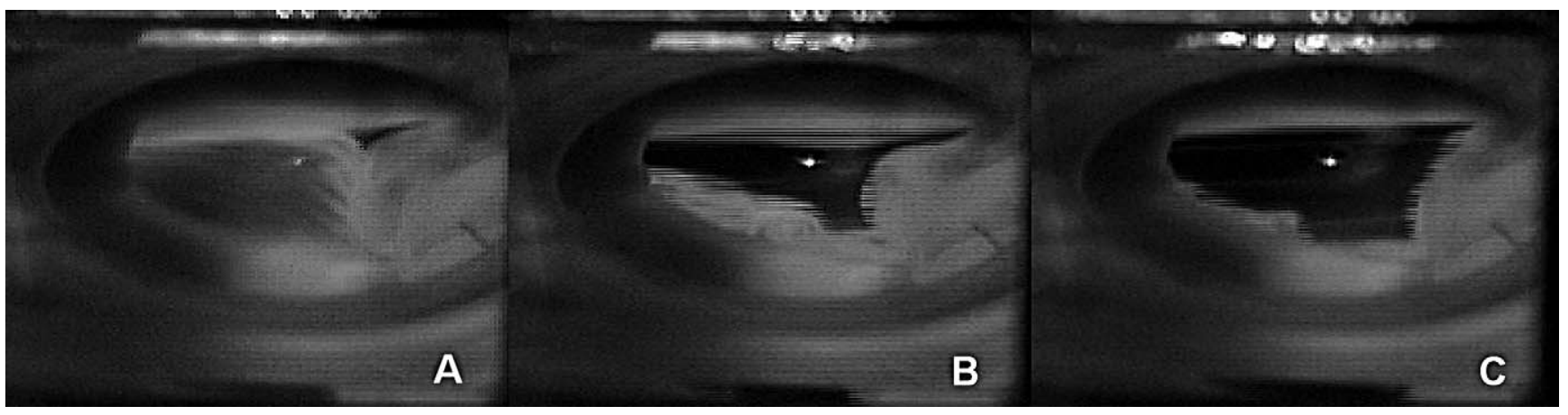

Figure 3. Stented heart valves in the bioreactor system with the valve in the closed (A), intermediate (B), and opened (C) positions.

\section{Phenotypic Cell Identification by Means of Immunocytochemistry}

ECs were identified by the presence of Factor VIII-related antigen (von Willebrand factor; DAKO Cytomation GmbH, Hamburg, Germany). MFs were characterized by means of incubation with monoclonal mouse antibodies for $\alpha$-smooth muscle actin (Sigma), desmin (DAKO), vimentin (DAKO), and fibronectin (DAKO).

\section{Biochemical Assays}

Biochemical assays were performed for analysis of cellular and extracellular components of native and decellularized porcine heart valves. For this purpose, an additional 8 valve leaflets for each assay were used. All samples were normalized according to equivalent dry weight. A DC Protein-Assay (Bio-Rad, Hercules, Calif) was used to detect the total protein concentration. Total DNA was isolated and purified by means of sequential organic extractions with phenol (Roth, Karlsruhe, Germany) and phenol-chloroform-isoamyl alcohol (Roth) and quantified by means of spectrophotometry (Helios $\beta$; Spectronic Unicam, Cambridge, United Kingdom). Cell proliferation was ascertained with a CellTiter Non-Radioactive Cell Proliferation Assay (Promega) and measured with the 96-well plate enzyme-linked immunosorbent assay reader "Sunrise" (TECAN, Crailsheim, Germany) for metabolic activity. Tissue was completely acid digested to measure the total 4-hydroxyproline content. After tissue extraction, total collagen, elastin, and glycosaminoglycan levels were quantified by using SIRCOL, FASTIN, and BLYSCAN assays (Biocolor, Belfast, Northern Ireland). ${ }^{8}$

\section{Histology}

Each heart valve was carefully removed from the stent, and the 3 leaflets were separated. One leaflet was processed for histology. Specimens were fixed for 72 hours at $20^{\circ} \mathrm{C}$ in $4 \%$ paraformaldehyde in $0.1 \mathrm{~mol} / \mathrm{L}$ cacodylate buffer, $\mathrm{pH}$ 7.4. They were washed in tap water for 2 hours, dehydrated in graded alcohol, and finally embedded in paraffin (Merck, Darmstadt, Germany). Sections (5-8 $\mu \mathrm{m}$ ) of each tissue sample were deparaffinized and rehydrated. For general morphology, serial sections were stained with hematoxylineosin stain (cellular components and nuclei). For identification of ECs on the tissue surface, Factor VIII-related antigen staining was conducted. The slides were thoroughly rinsed, with repeated turn- ing of the slide during staining, to avoid edge-effect artifacts. Sections were analyzed and documented by using common brightfield light microscopy (Axiovert S 100; Zeiss, Jena, Germany).

\section{Environmental Surface Electron Microscopy}

The remaining 2 leaflet specimens for environmental surface electron microscopy were fixed in $4 \%$ paraformaldehyde for 24 hours. Fixed specimens were dehydrated in an alcohol gradient (40\%, $60 \%, 80 \%$, and $99.9 \%$ for 10 minutes each). Samples were critical point dried by using a critical point drier (CPD030; BAL-TEC, Balzers, Liechtenstein) and mounted on stubs for surface electron microscopy. Mounted samples were subsequently coated with gold by using a sputter coating device SCD005 (BAL-TEC). Samples were analyzed with a scanning electron microscope (LEO-1450 VP; LEO, Oberkochen, Germany).

\section{Statistical Analysis}

All results are presented as mean values \pm standard deviations (95\% confidence intervals). The 2 -sided $t$ test was used to assess differences between the heart valve tissues. Statistical analysis was conducted with the software package SPSS for windows, version 10.0 (SPSS GmbH Software, München, Germany).

\section{Results}

\section{Isolated ECs and MFs}

The confluent ECs displayed a cobblestone morphology of flattened cells with large rounded nuclei and demonstrated strong granular perinuclear immunofluorescence for von Willebrand factor (data not shown). Immunocytochemistry of isolated and fixed carotid artery medial cells revealed a strong expression of $\alpha$-smooth muscle actin, vimentin, and fibronectin, which proved the MF phenotype, according to previous publications. ${ }^{4,9}$

\section{Decellularized Matrix}

Histologic analysis of the decellularized valve tissue sections stained with hematoxylin and eosin showed that treatment with trypsin-EDTA for 12 hours followed by a 24- 


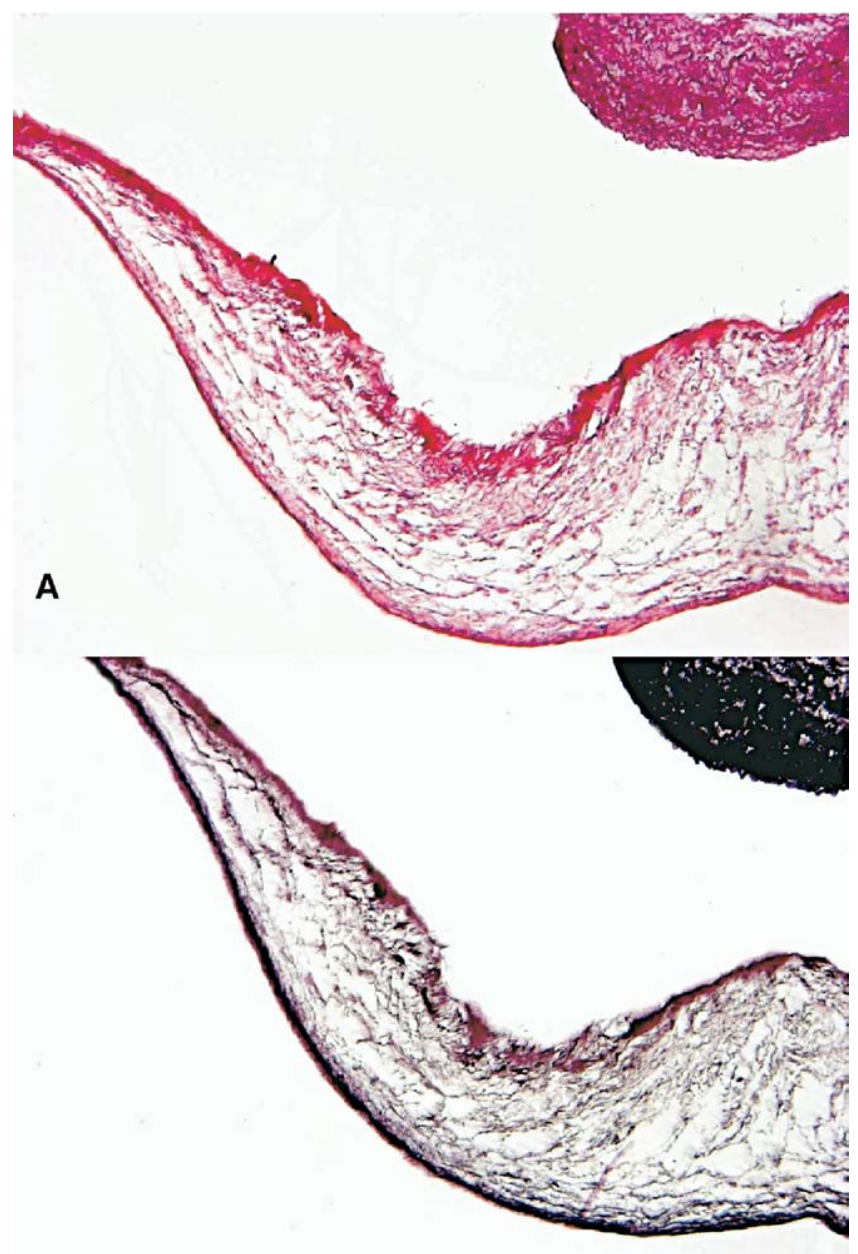

B

Figure 4. Hematoxylin-eosin (A) and 4',6-diamidino-Z-phenylindole $(B)$ staining reveal the ingrowth of ovine myofibroblasts into the valve leaflets, resulting in a tissue comparable with a native pulmonary heart valve in both groups. (Original magnification $200 \times$.)

hour washing with PBS converted native porcine pulmonary valves in a cell-free scaffold (Figure 4, A). A Russel-Movat pentachrome stain visualized the physiologic valve structure consisting of arterialis, fibrosa, spongiosa, and ventricularis, with no apparent disruptions of the leaflet histoarchitecture (Figure 4, B). DNA and proliferation assays showed amounts of cells and cell debris (41.06 \pm 5.5 vs $6.83 \pm 2.31$ $\mu \mathrm{g} / \mathrm{mg}$ dry tissue; $95 \%$ confidence interval, 29.65 respective $38.79 ; P<.05)$, corresponding to an $84 \%$ reduction of cellular components (each $n=8$ ). As confirmed by means of histology, the remaining DNA does not resemble intact cells but rather cell debris. Assays for quantification of extracellular matrix demonstrated a good preservation of the major structural components (collagen, elastin, and glycos-

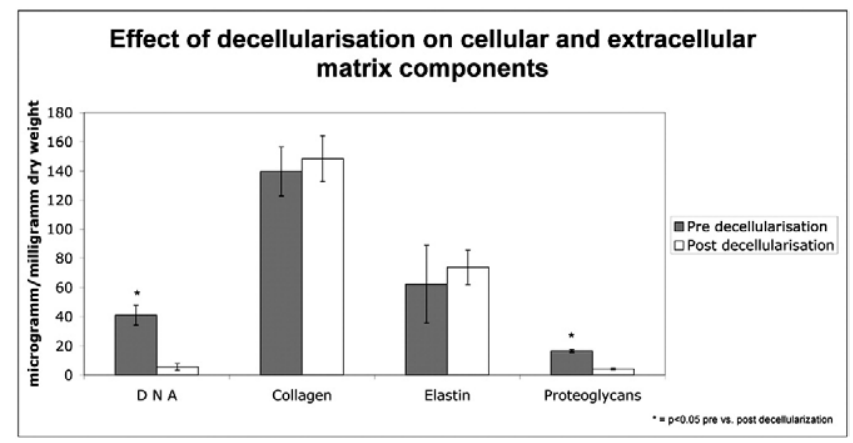

Figure 5. Effect of decellularization on cellular and extracellular matrix components.

aminoglycans; Figure 5). The detected minor increase of collagen and elastin after the decellularization process is an already described phenomen ${ }^{10}$ caused by the use of trypsinEDTA, inducing an enhanced collagen and elastin extraction effect on the matrix.

\section{Macroscopic Appearance of Constructs After Deployment}

The hydrodynamic pulsatile flow system enabled synchronous opening and closing of the leaflets throughout the entire culture period in all tissue-engineered stent valves. After folding and deployment, the valves were placed in the bioreactor. By using PBS medium, they were tested for competent opening and closing. Gross appearance showed intact and pliable constructs.

\section{Histology and Immunohistochemistry of Reseeded Valves}

Hematoxylin-eosin staining revealed a confluent ingrowth of ovine MFs into the valve leaflets, revealing a picture comparable with that of native tissue in both groups (Figure 6). From a recent study, we know that this ingrowth is uniform from the inflow and outflow side of the valve leaflets. ${ }^{4}$ Surface characteristics differed significantly. In the out-stent group almost no surface standing cells were identified. In the in-stent group contrast-flattened cells, resembling ECs, covered the leaflet structures (Figure 7, A). Immunohistochemistry with Factor VIII-related antigen confirmed this observation (Figure 7, B).

\section{Environmental Surface Electron Microscopy}

Samples $(\mathrm{n}=3)$ of valve leaflets after in vitro culture and before and after folding and deployment were compared. The pretreatment group revealed a smooth surface without cell and tissue disruption (Figure 8,A). The in-stent group showed a similar appearance with minimal injury (Figure 8, $B)$. In contrast, the out-stent group had severe tissue damage, with matrix disruption and deep lesions (Figure 8,C). 


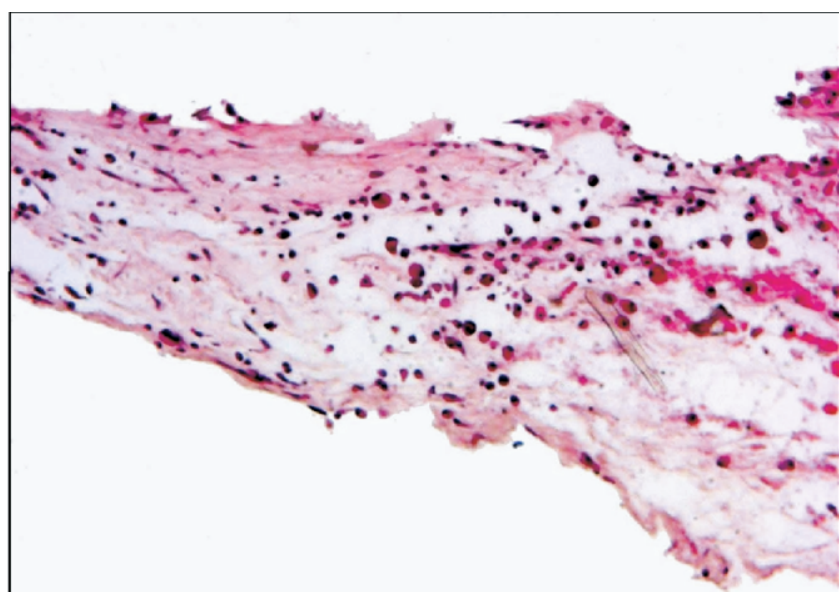

A

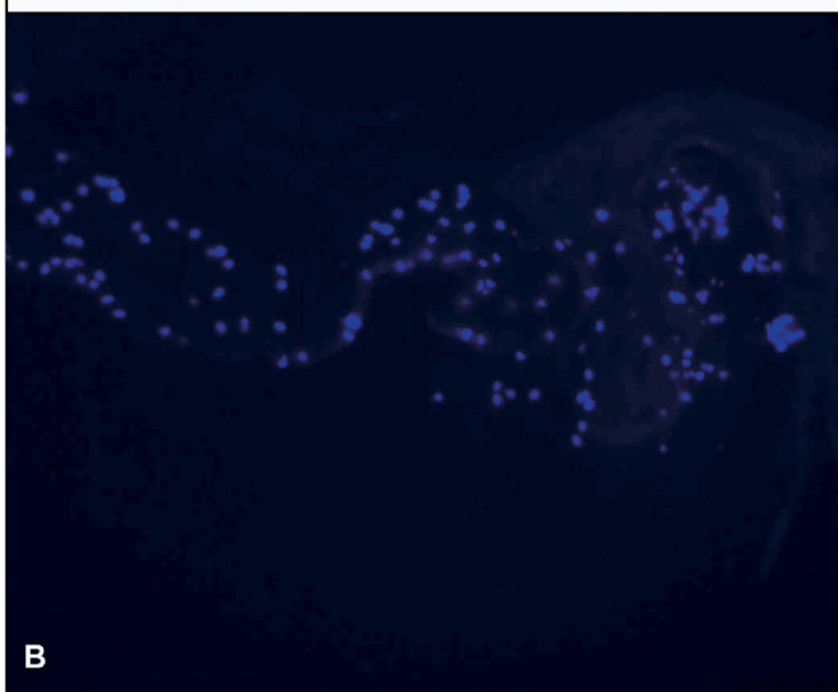

Figure 6. Hematoxylin-eosin staining reveals the ingrowth of ovine myofibroblasts into the valve leaflets, resulting in a tissue comparable with a native pulmonary heart valve in both groups. (Original magnification 200×.)

\section{Discussion}

Right ventricular dysfunction caused by severe pulmonary valve regurgitation is a serious complication after transannular patch augmentation of the RVOT for treatment of tetralogy of Fallot. ${ }^{11}$ Up to 5 years ago, the treatment of choice was a redo operation with reconstruction of the RVOT with a valved conduit. ${ }^{12}$ After extensive in vivo evaluation, ${ }^{13}$ Bonhoeffer and colleagues ${ }^{14}$ introduced the concept of endovascular pulmonary valve application clinically. Because of the limited diameter of the vascular access, they used low-profile, glutaraldehyde-fixed bovine jugular vein valves. However, these valves, like all biologic valves, are likely prone to degeneration and calcification and have a limited long-term function. A recent publication

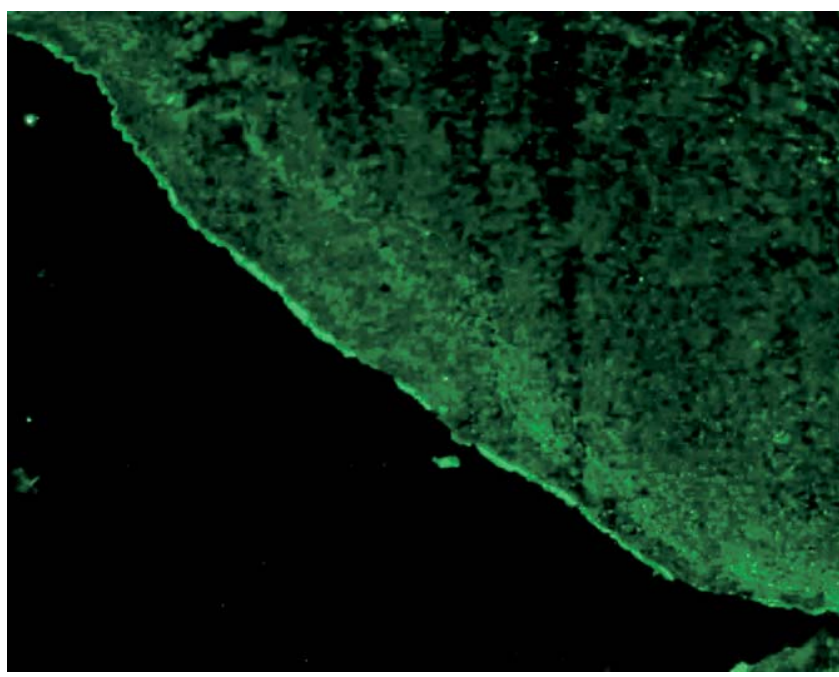

Figure 7. Immunohistochemistry with Factor VIII-related antigen confirmed the identity of the flattened surface-located cells as endothelial cells. (Original magnification $400 \times$.)

of Ruiz and associates ${ }^{15}$ investigated the use of acellular SIS to create a low-profile heart valve for transcatheter placement by using a guided tissue regeneration approach. Guided tissue regeneration differs from tissue engineering by using acellular matrices that ought to be repopulated after implantation by the adjacent host tissue. ${ }^{16}$ Although the results of Ruiz and associates ${ }^{15}$ indicate degradation of the SIS with some kind of remodeling by means of tissue neoformation, it is unlikely that the obtained results can be transferred to human subjects. ${ }^{17,18}$ Accordingly, we believe that preimplantation seeding by means of tissue engineering remains a prerequisite. In a recent publication the complete repopulation of a decellularized porcine pulmonary heart valve with ovine vascular wall cells was demonstrated. ${ }^{4}$ Complete pulmonary roots will require large deployment catheters $(>24 \mathrm{~F})$. Because these will potentially jeopardize the access vessel (femoral vein), we decided to reduce the valve dimensions by removing the sinus and the majority of the muscular annulus. Placement of the now scalloped heart valve leaflets in a stent alone will, however, have only a very small attachment area in the patient's pulmonary root, and paravalvular leakage is very likely to occur. A wrapping cuff with SIS was applied to prevent these leaks and facilitate cell seeding (in a scalloped valve the cell suspension will rinse off immediately). We have now shown that the location of the SIS wrap is crucial to prevent injuries of the nitinol struts during folding and deployment. Only if the SIS is implanted between the stent and the scalloped valve leaflets (outflow surface) can it protect the valve leaflets from device-related trauma. A remaining limitation is the fact that the deployment catheter consists of on outer sheath 


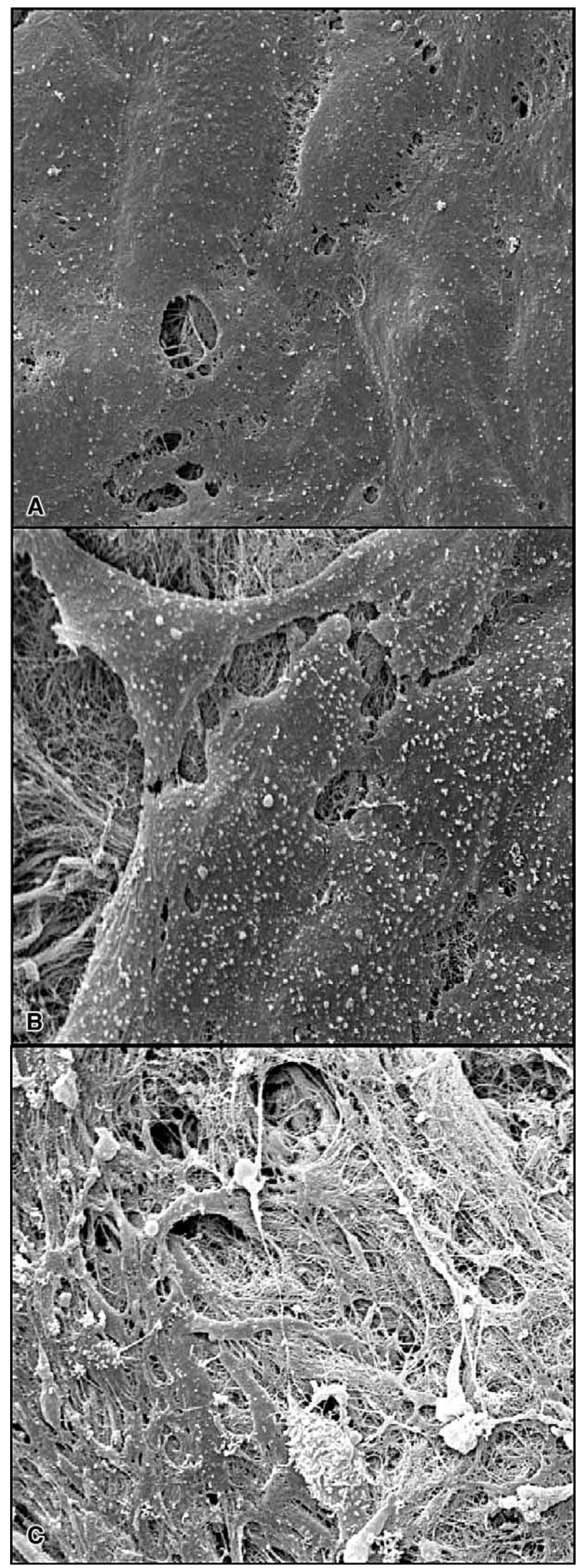

and a central mandrill. This mandrill passes through the valve and causes trauma to the inflow area of the valve leaflets. This is a mechanism well known from transvalvular catheter procedures. We believe that the endothelial abrasions require further in-depth studies, with particular focus on in vivo studies.

From clinical experience, the RVOT in patients after repair of tetralogy of Fallot is normally not tube like but rather funnel like. ${ }^{11}$ Proximal anchoring of the stent in the RVOT is crucial because migration will result in valvular dysfunction. New stents are currently under testing to offer individually customized stent solutions for patients. ${ }^{19,20}$ The applied SIS is very compliant and can be easily modeled to basically all stent designs.

A variety of limitations remain to be addressed. In addition to the evaluation of the optimal cell source for seeding of the constructs, ${ }^{9}$ the potential immunologic barrier of porcine extracellular matrix tissue in human subjects needs further clarification. ${ }^{21}$ An important and crucial limitation of midterm to long-term tissue engineering in general is an immanent infection risk. Despite the use of antibiotics in the tissue-culture medium, we observed bacterial or fungal infections during the initial 16 days of valve culture in more than $50 \%$ of the cases. All attempts to conduct long-term testing ( $>24$ hours) after the final deployment maneuver failed because of bacterial or fungal infections $(n=9)$. We believe that this can be attributed to 2 circumstances. First, the repeated manipulation (implantation in the bioreactor, dynamic culture, EC seeding, removal from the bioreactor with subsequent folding, and deployment and repeated testing in the bioreactor) bears an infection risk. Second, the used porcine hearts and SIS, as well as the ovine carotid artery segments, were obtained from a local slaughterhouse under "clean" conditions. Sole use of sterilely obtained hearts, SIS, and arteries for in vitro testing was turned down from our local animal ethics committee. To overcome this limitation, we are currently testing the tissue-engineered constructs in the pulmonary valve position of growing sheep.

\section{Conclusion}

In the present study we demonstrated for the first time the successful merging of 2 novel technologies: endovascular valve application and tissue engineering. Furthermore, the critical issue of device-related tissue damage during valve folding and deployment was successfully addressed. The resulting necessity of in vivo testing is currently being

Figure 8. Environmental surface electron microscopy. A, The control group (native ovine pulmonary valve) revealed a smooth surface without cell and tissue disruption. B, The in-stent group showed a similar appearance with minimal injury. C, In contrast, the out-stent group had severe tissue damage, with matrix disruption and deep lesions. 
addressed in a study using the engineered heart valve constructs in the pulmonary roots of juvenile sheep.

\section{References}

1. de Ruijter FT, Weenink I, Hitchcock FJ, Meijboom EJ, Bennink GB. Right ventricular dysfunction and pulmonary valve replacement after correction of tetralogy of Fallot. Ann Thorac Surg. 2002;73:1794-800.

2. Coats L, Tsang V, Khambadkone S, van Doorn C, et al. The potential impact of percutaneous pulmonary valve stent implantation on right ventricular outflow tract re-intervention. Eur J Cardiothorac Surg. 2005;27:536-43.

3. Nerem RM. Cellular engineering. Ann Biomed Eng. 1991;19:529-45.

4. Schenke-Layland K, Opitz F, Gross M, et al. Complete dynamic repopulation of decellularized heart valves by application of defined physical signals—an in vitro study. Cardiovasc Res. 2003;60:497-509.

5. Hodde JP, Record RD, Tullius RS, Badylak SF. Retention of endothelial cell adherence to porcine-derived extracellular matrix after disinfection and sterilization. Tissue Eng. 2002;8:225-34.

6. Shell DH 4th, Croce MA, Cagiannos C, et al. Comparison of smallintestinal submucosa and expanded polytetrafluoroethylene as a vascular conduit in the presence of gram-positive contamination. Ann Surg. 2005;241:995-1001.

7. White JK, Agnihotri AK, Titus JS, Torchiana DF. A stentless trileaflet valve from a sheet of decellularized porcine small intestinal submucosa. Ann Thorac Surg. 2005;80:704-7.

8. Stock UA, Wiederschain D, Kilroy SM, et al. Dynamics of extracellular matrix production and turnover in tissue engineered cardiovascular structures. J Cell Biochem. 2001;81:220-8.

9. Schenke-Layland K, Riemann I, Opitz F, et al. Comparative study of cellular and extracellular matrix composition of native and tissue engineered heart valves. Matrix Biol. 2004;23:113-25.

10. Schenke-Layland K, Vasilevski O, Opitz F, et al. Impact of decellularization of xenogeneic tissue on extracellular matrix integrity for tissue engineering of heart valves. J Struct Biol. 2003;143:201-8.
11. Frigiola A, Redington AN, Cullen S, Vogel M. Pulmonary regurgitation is an important determinant of right ventricular contractile dysfunction in patients with surgically repaired tetralogy of Fallot. Circulation. 2004;110(suppl):II153-7.

12. Mayer JE Jr. Uses of homograft conduits for right ventricle to pulmonary artery connections in the neonatal period. Semin Thorac Cardiovasc Surg. 1995;7:130-2.

13. Bonhoeffer P, Boudjemline Y, Saliba Z, et al. Transcatheter implantation of a bovine valve in pulmonary position. Circulation. 2000;102: 813-6.

14. Bonhoeffer P, Boudjemline Y, Saliba Z, et al. Percutaneous replacement of pulmonary valve in a right-ventricle to pulmonary-artery prosthetic conduit with valve dysfunction. Lancet. 2000;356:1403-5.

15. Ruiz CE, Iemura M, Medie S, et al. Transcatheter placement of a low-profile biodegradable pulmonary valve made of small intestinal submucosa: a long-term study in a swine model. J Thorac Cardiovasc Surg. 2005;130:477-84.

16. Stock UA, Schenke-Layland K. Performance of decellularized xenogeneic tissue in heart valve replacement. Biomaterials. 2006;27:1-2.

17. Simon P, Kasimir MT, Seebacher G, et al. Early failure of the tissue engineered porcine heart valve SYNERGRAFT in pediatric patients. Eur J Cardiothorac Surg. 2003;23:1002-6.

18. Goldstein S, Clarke DR, Walsh SP, et al. transpecies heart valve transplant: advanced studies of a bioengineered xeno-autograft. Ann Thorac Surg. 2000;70:1962-9.

19. Migliavacca F, Schievano S, Dubini G, et al. Percutaneous valve replacement: an engineer's perspective. Presented at: Advances in Tissue Engineering and Biology of Heart Valves Meeting; Florence, Italy; Sept 15-18, 2004.

20. Lutter G, Kuklinski D, Berg G, et al. Percutaneous aortic valve replacement: an experimental study. I. studies on implantation. $J$ Thorac Cardiovasc Surg. 2002;123:768-76.

21. Rieder E, Seebacher G, Kasimir MT, et al. Tissue engineering of heart valves: decellularized porcine and human valve scaffolds differ importantly in residual potential to attract monocytic cells. Circulation. 2005;111:2792-7. 